\title{
Heterotopic tubal pregnancy with live twin birth complicating ovulation induction assisted cycle
}

\author{
Adebiyi Gbadebo Adesiyun ${ }^{1 *}$, Benson Ayodele-Cole ${ }^{2}$ \\ ${ }^{1}$ Department of Obstetrics \& Gynaecology, Ahmadu Bello University Teaching Hospital, Zaria, Nigeria \\ ${ }^{2}$ Echo-Scan Services, Kaduna, Nigeria \\ Email: "biyi.adesiyun@yahoo.com
}

Received 10 April 2012; revised 14 May 2012; accepted 31 May 2012

\begin{abstract}
Heterotopic pregnancy is an uncommon clinical entity in Nigeria even though some of the risk factors are highly prevalent in the population. We report a case of heterotopic pregnancy following ovulation induction with clomiphene citrate. A favourable outcome resulting in live twin birth was recorded despite the presence of poor clinical features that affects pregnancy and foetal outcome.
\end{abstract}

Keywords: Heterotopic Pregnancy; Ovulation Induction; Clomiphene Citrate; Laparotomy; Twin Birth

\section{INTRODUCTION}

Heterotopic pregnancy is said to occur when there is simultaneous coexistence of two or more intrauterine and extra uterine implantation sites. Heterotopic pregnancy could follow an assisted conception cycle or a natural conception cycle. Interestingly, there has been an increase in the incidence of assisted conception related heterotopic pregnancy since the introduction of assisted conception technology into the treatment array of infertile couples [1]. Heterotopic pregnancy is an uncommon clinical condition in Nigeria probably due to underreporting or missed diagnosis, because the risk factors like pelvic inflammatory disease, ectopic pregnancy and multiple pregnancy are highly prevalent in the population [2]. We report a case of heterotopic tubal pregnancy following ovulation induction with clomiphene citrate, resulting in twin live birth.

\section{CASE REPORT}

A 36-year old Nigerian woman presented with a year history of infertility. She was para $1+1$. Her last child birth was 23 months prior to presentation; she had a vaginal delivery of a live female baby and the placenta

\footnotetext{
*Corresponding author.
}

was manually removed under anaesthesia. She had stopped breastfeeding 11 months earlier. Evaluation revealed a normal pelvic ultrasound scan, serum prolactin of 26.2 $\mathrm{ng} / \mathrm{ml}$, normal semen analysis and the hysterosalpingography showed a normal size uterine cavity, left cornual tubal occlusion and right fimbrial end tubal occlusion. She was commenced on treatment with cabergoline and had therapeutic hydrotubation. After a month treatment with cabergoline, a repeat serum prolactin was $12.3 \mathrm{ng} / \mathrm{ml}$. She subsequently had ovulation induction with clomiphene citrate, followed with follicular tracking and timed intercourse after human chorionic gonadotrophin injecttion was given whenever the lead follicle was at least 18 $\mathrm{mm}$. She conceived at the fourth assisted conception treatment course with the last menstrual period being on the $13^{\text {th }}$ of December, 2010. Pregnancy was confirmed with a positive serum human chorionic gonadotrophin hormone and an ultrasound scan that showed two viable intrauterine fetuses at gestational age of 6 weeks and 4 days.

She suddenly felt a sharp lower abdominal pain when the pregnancy was 7 weeks, the pain progressed and necessitated her to present in the hospital the next day. Assessment revealed severe pallor, signs of hypovolemia and features of intra abdominal fluid collection. A diagnosis of ruptured ectopic pregnancy was made. An urgent sonography revealed twin viable intrauterine fetuses and ruptured adnexial ectopic pregnancy, thus confirming the diagnosis of heterotopic pregnancy. She was resuscitated and had an urgent laparotomy. Intra operative findings were haemoperitoneum of 2 litres and a ruptured right ampullary tubal ectopic pregnancy; she had right salpingectomy. In all she had 4 units of whole blood transfused and post operative treatment include antibiotics, analgesia and progesterone injection. Post-operative period was uneventful. Sonography done on $7^{\text {th }}$ day post surgery showed viable intrauterine twin fetuses.

Thereafter pregnancy remained uneventful until 36 weeks when she had spontaneous rupture of fetal membranes. She had emergency caesarean section because 
the fetuses were lying transversely. She was delivered of 2 male fetuses that weighed $2.5 \mathrm{~kg}$ and $2.05 \mathrm{~kg}$ with good apgar scores. She had primary postpartum haemorrhage due to uterine atony and lost about 2.2 litres of blood despite rectal insertion of misoprostol, manual uterine compression .and oxytocin drip. She had a total of 5 units of blood transfusion intra and post operatively. On the $8^{\text {th }}$ post surgery day she was discharged home following good recuperation and satisfactory general condition.

\section{DISCUSSION}

Heterotopic pregnancy which was first described at an autopsy study [3] was hitherto a rarity before the introduction and widespread use of ART. In natural conception cycles low frequency ranging from 1 in 10,000 to 1 in 50,000 was reported and high rates of heterotopic pregnancy from 1 in 100 to 1 in 500 was reported in ART treatment cycles [4-6]. The later is attributable to super ovulation induction, multiple embryo transfer with resultant increase in multiple pregnancy and ectopic pregnancy. In this report the intrauterine pregnancy was a twin. This is in conformity with authors that reported an increase in and not in uterine twin gestation. In ARTrelated heterotopic pregnancy and an exclusive singleton intrauterine gestations in natural cycle related heterotopic pregnancy [4]. In this case report, heterotopic diagnosis was made at about seven weeks of gestation. This is within the $5^{\text {th }}$ to $8^{\text {th }}$ week gestation reported to be the commonest diagnostic period interval for heterotopic pregnancies [4,7]. Furthermore, the type of cycle, be it assisted or natural cycle did not affect the gestational age at diagnosis [4]. The ectopic gestation in this report was tubal, similar to finding in other series $[4,7,8]$. Though, the ectopic gestation was in the right tube in this patient, there is no predilection for right or left tube in heterotopic pregnancy $[4,8]$. In the evaluation of this patient before index conception, she had hysterosalpingographic tubal occlusion and therapeutic hydrotubation as earlier described by these authors [9]. Studies have highlighted the importance of previous pelvic pathology as a risk factor for heterotopic pregnancy, especially in the cohort that had heterotopic pregnancy following assisted conception [4].

The clinical presentation in this case report includes hypovolemic shock, tubal rupture and haemoperitoneum. Being a case of heterotopic pregnancy following assisted conception this finding are in contrast to that of a series that reported on outcome of heterotopic pregnancies and found a significantly lower rate of low systolic blood pressure, tubal rupture and haemoperitoneum in the assisted conception group compared to spontaneous conception group [4]. A high miscarriage rate have been reported in multiple pregnancy and pregnancy complicated by heterotopic implantation with higher rate of miscarriage documented in heterotopic pregnancy complicating assisted conception than spontaneous conception [4,10-12]. The aggregate of these predisposing factors did not result in miscarriage in the case presented. Multiple pregnancy and Heterotopic pregnancy are known to individually predispose to preterm births $[4,7,13]$. This patient was delivered of 2 male babies by caesarean section at 36 weeks due to preterm spontaneous rupture of fetal membranes. In heterotopic pregnancy, survival rate of the intrauterine gestation range from $35 \%$ to $66.2 \%[7,14,15]$. This rates are influenced by variables that hovers round early diagnosis and treatment viz-a-viz treatment before tubal rupture and development of haemoperitoneum and hypovolemic shock that may result in hypoxic challenge to the intrauterine fetus [4]. A high survival rate translate to improve live birth rate. The poor clinical condition in this case as exhibited by tubal rupture, haemoperitoneum and shock did not affect the survival of the two fetuses which is in accord with another study that did not demonstrate a relationship between intra uterine fetal mortality and presence of haemoperitoneum [7]. Though with figure that was not statistically significant, live birth rate was reported to be two-fold higher in the subgroup that had heterotopic pregnancy following assisted conception compared to spontaneous conception [4]. Although, studies have not demonstrated a link between heterotopic pregnancy and fetal congenital anomalies, one of the twin in this report had an upper respiratory tract obstructive pathology with no definitive diagnosis up till the time of this report $[4,7]$. In conclusion, this report recorded a favourable fetal outcome.

\section{REFERENCES}

[1] Lemus, J.F. (2000) Ectopic pregnancy: An update. Current Opinion in Obstetrics and Gynecology, 12, 369-375. doi:10.1097/00001703-200010000-00005

[2] Eigbefoh, J.O., Mabayoje, P.S. and Aliyu, J.A.Y. (2008) Heterotopic pregnancy: A report of two cases. Nigerian Journal of Clinical Practice, 11, 85-87.

[3] Bright, D.A. and Gaupp, F.B. (1990) Hetertopic pregnancy: A reevaluation. The Journal of the American Board of Family Medicine, 3, 125-128.

[4] Han, S.H., Jee, B.C., Suh, C.S., Kim, S.H., Choi, Y.M., Kim, J.G. and Moon, S.Y. (2007) Clinical outcomes of tubal heterotopic pregnancy, assisted vs spontaneous conceptions. Gynecologic and Obstetric Investigation, 64, 49-54.

[5] Dor, J., Seidman, D.S., Levran, D., Ben-Rafael, Z., Ben Shlomo, I. and Mashiach, S. (1991) The incidence of combined intrauterine and extrauterine pregnancy after in vitro fertilization and embryo transfer. Fertility and 
Sterility, 55, 833-834.

[6] Marcus, S.F., Macnamee, M. and Brinsden, P. (1995) Heterotopic pregnancies after in-vitro fertilization and embryo transfer. Human Reproduction, 10, 1232-1236. doi,10.1093/humrep/10.1.199

[7] Tal, J., Haddad, S., Gordon, N. and Timor-Tritsch, I. (1996) Heterotopic pregnancy after ovulation induction and assisted reproductive technology, a literature review from 1971 to 1993. Fertility and Sterility, 66, 1-12.

[8] Reeece, E.A., Petrie, R.H., Sirmans, M.F., Finster, M. and Todd, W.D. (1983) Combined intrauterine and extrauterine gestations, a review. American Journal of Obstetrics \& Gynecology, 146, 323-330.

[9] Adesiyun, A.G., Cole, G. and Ogwuche, P. (2009) Hydrotubation in the management of female infertility, outcome in low resource setting. East African Medical Journal, 86, 31-36. doi,10.4314/eamj.v86i1.46925

[10] Seoud, M.A. (1992) Outcome of twin, triplet and quadruplet in in-vitro fertilization pregnancies, the Norfolk experience. Fertility and Sterility, 57, 825-834.
[11] Mcdonald, S., Murphy, K., Beyene, J. and Ohlsson, A (2005) Perinatal outcomes of in vitro fertilization twins, a systematic review and meta-analyses. American Journal of Obstetrics \& Gynecology, 193, 141-152. doi,10.1016/j.ajog.2004.11.064

[12] Goleman, G.A., Fisch, B., Ovadia, J. and Tadir, Y. (1992) Heterotopic pregnancies after assisted reproductive technologies. Obstetrical \& Gynecological Survey, 47, $217-$ 221. doi,10.1097/00006254-199204000-00001

[13] Talo, C.P. (1995) Maternal and neonatal morbidity associated with in vitro fertilization. The Journal of Pediatrics, 5, 794-800. doi,10.1016/S0022-3476(95)70175-3

[14] Smith, D.J.H. and Siddique, F.H. (1970) A case of heterotopic pregnancy. American Journal of Obstetrics \& Gynecology, 108, 1289-1290.

[15] Rojansky, N. and Schenker, J.G. (1996) Heterotopic pregnancy and assisted reproduction-An update. Journal of Assisted Reproduction and Genetics, 13, 594-601. doi:10.1007/BF02066615 